\title{
RECONSTRUÇÃO COM PRÓTESE IMEDIATA PÓS MAXILECTOMIA
}

\section{IMMEDIATE RECONSTRUCTION WITH PROSTHESIS FOLLOWING MAXILLECTOMY}

\author{
Renato Alberto Aldo Miracca; Josias de Andrade Sobrinho, ECBC-SP; \\ Antônio José Gonçalves, TCBC-SP
}

\begin{abstract}
RESUMO: Objetivo: Avaliar a eficiência da reconstrução com Prótese Imediata para Grandes Perdas da Maxila (PIGPM) em pacientes submetidos a maxilectomia, considerando-se o tempo de: internação hospitalar, retirada da sonda naso enteral, deglutição, fonação e reabilitação estética e funcional. Método: Foram avaliados 20 pacientes, 14 do sexo masculino, 6 do feminino com idade variando de 7 a 78 anos; 11 com Carcinoma Espino Celular (CEC). Realizamos a moldagem no intraoperatório com moldeira de acrílico com godiva termo-plástica ou alginato de sódio. O tempo de confecção da prótese foi de 45 minutos e todos os pacientes sairam da sala de cirurgia com a prótese instalada. Resultados: Os resultados foram satisfatórios nos 20 casos. Média: de internação 8,1 dias; retirada da sonda naso-enteral de 7,3 dias. A maioria dos pacientes foi alimentada por via oral em 3,3 dias, com alimentação pastosa. A voz foi considerada satisfatória. Conclusão: Concluímos que a PIGPM pós maxilectomia reduz o tempo para retirada da sonda naso enteral e o tempo de internação hospitalar; possibilita a alimentação precoce e melhora as condições da fala; reduz a deformidade provocada pela retração cicatricial; reintegra precocemente o paciente às suas atividades profissionais e sociais devendo ser adotada como rotina (Rev. Col. Bras. Cir. 2007; 34(5): 297-302).
\end{abstract}

Descritores: Neoplasias bucais; Prótese maxilo facial; Prótese total imediata.

\section{INTRODUÇÃO}

A cirurgia oncológica é responsável pelas grandes mutilações do maciço facial, cuja ressecção é imposta pelo tratamento. Estas ressecções podem ser parciais ou totais, uni ou bilaterais incluindo o maxilar, assoalho da órbita, globo ocular e apresentar comprometimento com o assoalho do crâneo.

A primeira maxilectomia parcial foi realizada Acoluthus e a primeira ressecção total foi praticada na França em 1827 por Gensoul. Possivelmente, Rogers já praticara ressecção quase total dos maxilares três anos antes, em New York ${ }^{1}$.

Considerado o pai da prótese buco maxilo facial Claude Martin, em 1889, foi primeiro a realizar uma prótese para maxilectomia ${ }^{2}$.

A reconstrução das perdas destas estruturas é complexa e, neste caso, os recursos da Prótese Imediata para Grandes Perdas do Maxilar (PIGPM) são da maior relevância. Em paises do $1^{\circ}$ mundo como no Reino Unido, $65 \%$ dos cirurgiões de cabeça e pescoço dispõem de um serviço de reabilitação com dentista especializado, mas não há referências a próteses realizadas no ato cirúrgico ${ }^{3}$. Em nosso país, poucos hospitais dispõem de uma equipe de cirurgiões de cabeça e pescoço que integre um serviço de reabilitação com dentista especializado para executar uma PIGPM, no ato de uma maxilectomia.

Alguns autores denominam Prótese Cirúrgica Imediata (PCI), uma prótese confeccionada sobre modelo de gesso do qual retirou-se a área delimitada pelo tumor. Esta prótese a que demoninamos Prótese Sobre Cirurgia de Modelos não requer a presença do Cirurgião Dentista na sala de cirurgia, nem de um Protético especializado, e tambem não elimina a necessidade do uso de tampões de gase na cavidade cirúrgica ${ }^{4}$.

As ressecções parciais ou totais da maxila acarretam grandes defeitos na boca e na face, sendo de importância funcional, psicológica e estética, a reabilitação protética pós maxilectomia. Esta reabilitação requer normalmente o uso de uma PIGPM, realizada imediatamente após a maxilectomia, seguida de uma prótese reparadora findo o período de cicatrização: aproximadamente quatro a oito semanas ${ }^{5-8}$.

Quando o paciente é portador de uma prótese, podemos utilizá-la adaptando-se a porção obturadora à mesma após a maxilectomia9.

Os cirurgiões de cabeça e pescoço, que não dispõe de um serviço especializado de prótese, recorrem aos tamponamentos da cavidade cirúrgica com gase furacinada

1. Cirurgião-Dentista; Mestre em Ciências da Saúde pelo Curso de Pós - Graduação em Ciências da Saúde do Hospital Heliópolis - Hosphel São Paulo SP; Assistente do Setor de Cirurgia Buco Maxilo Facial da Santa Casa de Misericórdia de São Paulo.

2. Doutor em Medicina pela UNIFESP; Professor Orientador do Curso de Pós- Graduação em Ciências da Saúde do Hospital Heliópolis - Hosphel. SP.

3. Mestre e Doutor pela Faculdade de Ciências Médicas da Santa Casa de São Paulo; Professor Adjunto - Chefe da Disciplina de Cirurgia de Cabeça e Pescoço da Faculdade de Ciências Médicas da Santa Casa de São Paulo.

Recebido em 23/01/2007

Aceito para publicação em 22/03/2007

Conflito de interesses: nenhum

Fonte de financiamento: nenhuma

Trabalho realizado na Disciplina de Cirurgia de Cabeça e Pescoço da Faculdade de Ciências Médicas da Santa Casa de São Paulo, Hospital Central da Irmandade da Santa Casa de Misericórdia de São Paulo.

Parte da Tese de Mestrado apresentada ao Curso de Pós Graduação em Ciências da Saúde do Hospital Heliópolis - Hosphel. SP. 
ou vaselinada, que necessitam trocas diárias. A retirada do tamponamento provoca : desagradável odor, conseqüência da fermentação dos fluidos na gase dentro da cavidade da maxila; grande sofrimento ao paciente pela manipulação da cavidade cruenta, além do risco de sangramento 9 .

Quando o paciente é enviado para realização da prótese, observa-se nas áreas de enxerto de pele e, especialmente, nas não enxertadas, grande deformidade facial e diminuição na abertura bucal, nestes casos, a prótese reparadora tardia, não recupera a deformidade cicatricial, não tem boa adaptação e em alguns casos é impossível de ser executada.

Nas crianças, uma equipe multidisciplinar que inclua o ortodontista é imprescindível, a fim de minimizar as deformações provocadas pela operação. A utilização de aparelhos ortodônticos que permitam adaptar uma prótese maxilar é extremamente útil pois possibilita o tratamento corretivo concomitante ao do câncer e, obtido o resultado esperado e terminada a fase de crescimento, é possível a realização de uma prótese reparadora para restabelecer a oclusão, a função mastigatória e a estética ${ }^{5-7}$.

A qualidade do tratamento e a reabilitação para doentes portadores de câncer de cabeça e pescoço, particularmente o paciente pediátrico, progrediu principalmente devido à preocupação com a integração das diversas especialidades envolvidas. Defeitos da cavidade oral, causados por trauma ou remoção de tecido maligno ou benigno exigem procedimentos especiais de tratamento. Além da radio e quimioterapia são também necessários: a fisioterapia e o acompanhamento psicológico do paciente e dos seus familiares. A higiene oral é essencial no processo de reabilitação. A integração multidisciplinar é mais eficiente, para o tratamento e recuperação dos pacientes ${ }^{5,7 ; 8-12}$.

A compensação da perda cirúrgica através de uma prótese obturadora é, geralmente, a solução mais adotada pela vantagem de não ser um procedimento invasivo e também por permitir, através da retirada da mesma, o exame clínico local para descoberta precoce de uma eventual recidiva ${ }^{13}$.

O grau de recuperação funcional de um paciente varia conforme as dimensões do defeito. O número e a posição dos dentes, as condições dentárias, a altura do rebordo alveolar residual, a presença ou não do assoalho palatino influem na estabilidade da prótese. A falta destes elementos torna a reabilitação dos pacientes edentados muito difícil ${ }^{5-11-14}$.

Para confecção de PIGPM destinada aos pacientes submetidos à maxilectomia foi descrita técnica de moldagem com material termoplástico (aquaplast), realizada no ato operatório sem necessidade do auxilio de técnico em prótese, fixada com amarrias ou parafusos corticais ${ }^{15}$. Tambem foram descritas técnicas de laboratório, em uma fase, que eliminam os procedimentos de moldagem, obtenção da relação cêntrica e da dimensão vertical, e utilizam as próteses do paciente, junto com a impressão funcional obtida ${ }^{9-16}$.

Prótese seccionada tripartida com ligações por magnetos pode ser executada,em casos de pouca abertura bucal, pois a porção do bulbo maxilar apresenta-se em partes menores, que podem ser introduzidas na boca com facilidade, e os magnetos promovem a união entre elas ${ }^{17-18-19}$. A utilização de magnetos e de silicone nos obturadores ocos seccionados para maxila possibilitam sua utilização em cavidades profundas. Uma armação de resina acrílica recoberta com uma camada fina de silicone previne a deformação da prótese obturadora $^{10,11,19-20}$.

Utilizando o espaço da abertura nasal, obtém-se um aumento da retenção das próteses obturadoras. O obturador é preparado de forma convencional ${ }^{21}$.

A literatura escassa sobre a PIGPM dificulta comparações estatísticas com outros autores e com outros procedimentos. O pouco que se encontra são relatos de casos com citações sobre a prótese, mas não avaliações com número significativo de casos submetidos à reconstrução com PIGP pós maxilectomias.

Em virtude da escassa literatura e pouca experiência sobre o assunto, propusemo-nos levantar nossa casuística, padronizar a técnica da prótese imediata e avaliar suas vantagens.

\section{MÉTODO}

Avaliamos os prontuários de 31 pacientes submetidos a maxilectomia pela Disciplina de Cirurgia de Cabeça e Pescoço da Santa Casa de Misericórdia de São Paulo, no período de 1991 a 2001. As próteses foram por nós executadas na área da Prótese Buco Maxilo Facial.

Quanto ao diagnóstico histopatológico, dos 31 casos, 26 foram neoplasias malignas, e 5 benignas. Das neoplasias malignas a de maior incidência foi Carcinoma Espino Celular (CEC) com 17 casos. O acesso a maxila foi realizado através da incisão de Weber Fergurson em 27 casos, enquanto o acesso intra-oral foi utilizado apenas em 3 casos.

Dos trinta e um pacientes com indicação para cirurgia com preparo de próteses, um paciente com a prótese preparada não foi operado, sendo enviado para tratamento paliativo; outro, após a ressecção do tumor, não apresentou apoio suficiente para a prótese, dois por complicações cirúrgicas, foram a óbito; um após a ressecção do tumor não apresentou comunicação com o seio maxilar, dispensando a prótese; dois prontuários omitiam dados relativos às próteses; um paciente recusou o uso da mesma. Em dois pacientes, por impossibilidade da ressecção total do tumor e terem margens comprometidas, não utilizamos as PIGPM, ficando as respectivas reabilitações para após a radioterapia com PGPM. Portanto nossa casuística consta de 20 casos.

A PIGPM é confeccionada de uma única forma em sua porção cavitária, variando somente na parte da arcada dentária, quando confeccionada precocemente. Já as PGPM foram confeccionadas de acordo com a extensão da operação, tamanho da cavidade a ser preenchida e a abertura bucal.

Nesta casuística foram executadas oito próteses imediatas simples ou inteiriças, cinco próteses bipartidas com ferrolho e sete próteses unidas por magnetos. Indicadas por aspectos estéticos e psicológicos, foram confeccionadas duaspróteses com dentes artificiais,. Não executamos PIGPM, conjugada com a prótese óculo-palpebral, dada a impossibilidade técnica para sua realização durante o tempo cirúrgico.

Foram avaliados os prontuários dos 20 pacientes submetidos a maxilectomia considerando: as datas de opera- 
ção e de alta hospitalar conseqüentemente o período de internação; a data da $1^{\circ}$ alimentação por via bucal; a dificuldade ou não durante a deglutição de alimento líquiido ou pastoso e a retirada da sonda naso enteral. A fonação, deglutição e a reabilitação estética foram por nós avaliadas por observação direta.

\section{Técnica da confecção da prótese}

A PIGPM pos maxilectomia obedeceu a um protocolo em três fases:

$1^{a}$ fase pré cirúrgica: avaliação do paciente com a equipe de cirurgia, moldagens e confecção de placas bases;

$2^{\mathrm{a}}$ moldagem durante o ato cirúrgico e confecção do bulbo maxilar; (Figura 1)

$3^{\text {a }}$ colocação, adaptação, orientação da higiene.

A reabilitação protética reparadora foi realizada após a cicatrização.

Quando as condições bucais e o tempo de execução permitiram, confeccionamos as próteses com dentes artificiais de acrílico. Isto traz ao paciente maior conforto psicológico e possibilidade de mastigação imediata.

Moldagem durante a cirurgia, confecção da porção maxilar.

Durante o ato cirúrgico, uma incisão de WeberFerguson é geralmente usada para acessar a lesão situada na maxila.

Retirado o tumor, realiza-se a moldagem da cavidade resultante, utilizando a base de acrílico e godiva termoplástica ou alginato de sódio. No transcorrer do ato cirúrgico, a ampliação das margens de ressecção podem obrigar mudanças no planejamento protético.

O tempo de execução total da prótese na fase laboratorial varia de 45 a 60 minutos, aproximadamente.

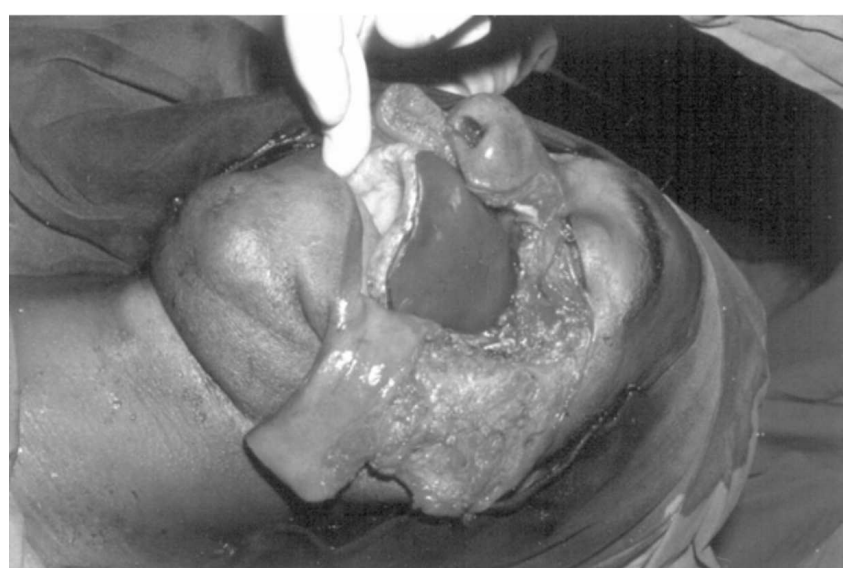

Figura 1 - Moldagem durante o ato cirurgico. Material utilizado termoplástico (godiva).
A peça protética pode ser inteiriça ou seccionada. Quando a cavidade bucal apresenta abertura suficiente, a prótese pode ser inteira, caso contrário, utiliza-se a prótese dividida em duas partes: a porção da cavidade maxilar e a porção da área do rebordo alveolar do maxilar, ambas unidas por magnetos ou fecho tipo ferrolho ${ }^{14}$. A prótese seccionada permite que pacientes com abertura bucal reduzida, possam colocar as suas peças com maior comodidade, porque a porção intra cavitária é posicionada em primeiro lugar e a porção da arcada dentária é posicionada, a seguir, fixando-se as duas através dos magnetos ou introduzindo-se a trava do ferrolho. (Figuras 2 e 3) ${ }^{19-23}$.

\section{Colocação, adaptação e orientação da higiene}

$O$ paciente deve ser informado das necessidades e procedimentos protéticos pós cirúrgicos e problemas associados à maxilectomia: dificuldades de deglutição, revisões da prótese obturadora, importância do acompanhamento durante o ajuste da prótese obturadora; especificamente problemas associados à fala e à nasalidade. No início, o paciente notará um pouco de vazamento de ar e de líquidos ao redor do bulbo da prótese obturadora.

A reabilitação protética permanente inicia-se com a completa cicatrização da ferida cirúrgica e assim que a sensibilidade do paciente permitir as moldagens. O processo e os materiais de moldagem são os mesmos descritos anteriormente, podendo-se fazer uso dos modelos arquivados para o preparo das moldeiras e bases.

Os pacientes classificados em três grupos, de acordo com a extensão da ferida cirúrgica: $1^{\circ}$ - submetidos somente à maxilectomia (14 pacientes); $2^{\circ}$ - os que, além da maxilectomia, realizaram a exenteração de órbita ( quatro pacientes ); e $3^{\circ}$-os que realizaram a maxilectomia, exenteração de órbita e craniotomia (dois pacientes), pois, o tempo de recuperação destes é maior que dos primeiros (Tabela 1).

\section{RESULTADO}

Em relação ao período de internação o resultado observado, em 20 pacientes, foi de 8,1 dias em média. Para o $1^{\circ}$ Grupo, 14 pacientes, a média foi cinco dias. Para o $2^{\circ}$ Grupo, quatro pacientes a média foi 6,25 dias. Para o $3^{\circ}$ Grupo, apenas dois pacientes, a média foi de 29 dias.

Em relação à sonda naso enteral, esta foi retirada em média com 7,3 dias após a cirurgia. No $1^{\mathrm{a}}$ Grupo, 14 pacientes, a média foi de 5,5 dias. Excluindo os dois pacientes em que a prótese ficou mal adaptada, esta média cai para 3,5 dias. No $2^{\circ}$ Grupo, quatro pacientes, a média foi de quatro dias. Para o $3^{\circ}$ Grupo, dois pacientes, a média foi de 34 dias.

Tabela 1 - Avaliação das próteses.

\begin{tabular}{|c|c|c|c|c|c|c|}
\hline Operação & Positivo & $\%$ & Negativo & $\%$ & Totais & $\%$ \\
\hline Maxilectomia & 13 & 65 & 1 & 5 & 14 & 75 \\
\hline Max. e Exent. de Órb. & 4 & 20 & 0 & 0 & 4 & 15 \\
\hline Máx. Exen. Órbita e Cran. & 1 & 5 & 1 & 5 & 2 & 10 \\
\hline Totais & 18 & 90 & 2 & 10 & 20 & 100 \\
\hline
\end{tabular}


Em relação à deglutição, os pacientes foram acompanhados pela equipe da fonoaudiologia, nos exercícios de deglutição de alimentos líquidos e pastosos e conseguiram realizar, sem dificuldade, as manobras aplicadas em 7,1 dias. Não considerando os dois pacientes que além da maxilectomia realizaram craniotomia e um paciente que teve a prótese mal adaptada, esta média caiu para 3,3 dias, apresentando alguma deficiência apenas com alimentos sólidos, pela ausência de dentes na PIGPM e pela sensibilidade da ferida cirúrgica recente. Este fato possibilitou a retirada da sonda naso enteral precocemente. Apenas um paciente teve maior dificuldade, apresentando refluxo nasal, superado após quatro dias graças ao empenho nos exercícios de deglutição.

Após este periodo de adaptação, todos os pacientes puderam alimentar-se normalmente, dispensando o uso da sonda naso enteral de alimentação.

Após a cicatrização da ferida cirúrgica, pequenos ajustes na prótese melhoraram a adaptação da mesma, assim como a alimentação dos pacientes.

Em relação à fonação, esta se apresentou inteligível, normal ou ligeiramente hipernasalada, em alguns casos.

Após a colocação da prótese permanente, tanto a deglutição quanto a fonação tiveram melhor desempenho. Esta avaliação foi feita por comparação com e sem o uso da prótese, não sendo possível aplicar qüestionários validados, para tal finalidade.

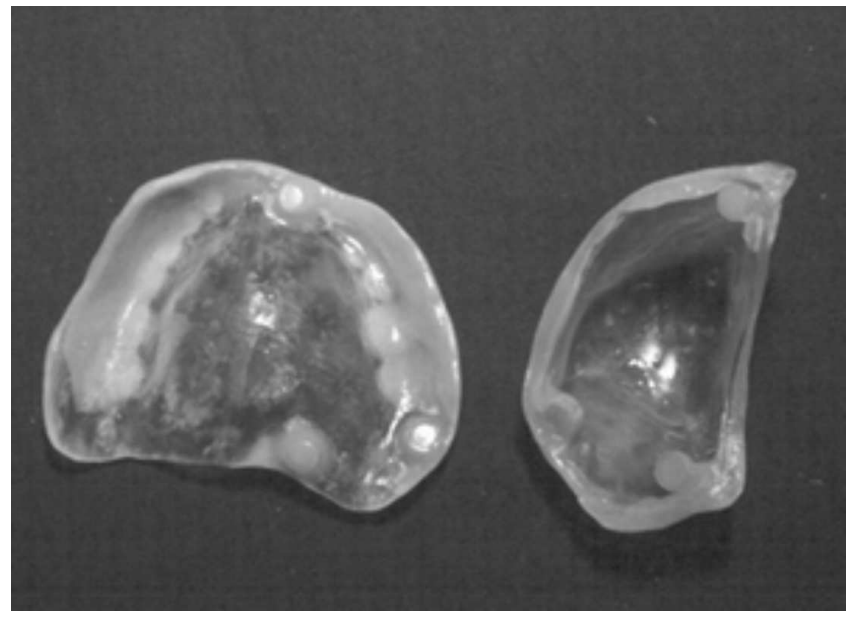

Figura 2 - Prótese Bi-partida com magnetos para sua união.

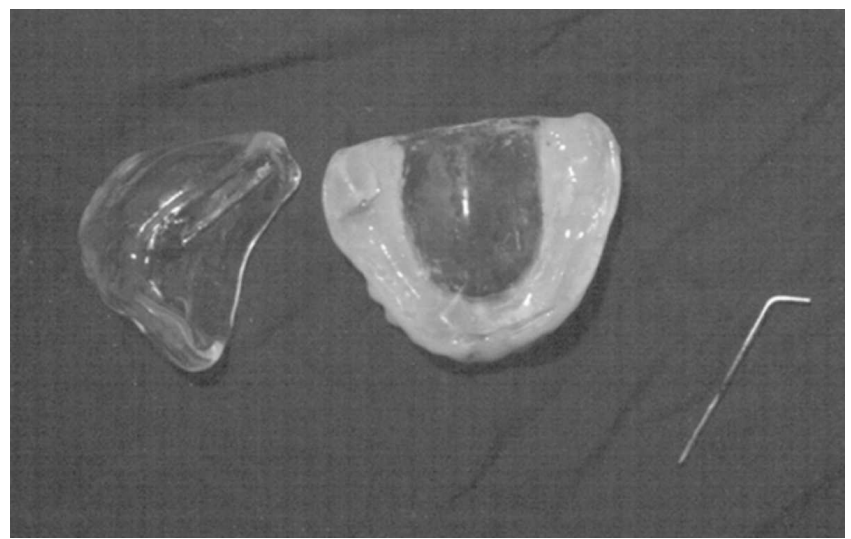

Figura 3 - Prótese Bi-partida com sistema de ferrolho para sua união.
Os tecidos suportados pela prótese mostraram-se bem posicionados, demonstrando uma retração cicatriciaal menor, como se verificou com o tempo.

\section{DISCUSSÃO}

As ressecções parciais ou totais da maxila acarretam grandes deformidades na boca e na face, sendo de importância funcional, psicológica e estética, a reabilitação protética pós maxilectomia. O uso de uma PIGPM reduz a retração cicatricial reduzindo a deformidade facial, facilitando a confecção da prótese permanente.

Pacientes submetidos à ressecção do maxilar sem a reabilitação com prótese obturadora, apresentam déficit na mastigação, na deglutição e apresentam também fonação anasalada, além de refluxo alimentar pela cavidade nasal. Este déficit, resultado de uma maxilectomia, é evitado ou reduzido pela reabilitação com PIGPM.

Encontramos, na literatura, trabalhos sobre a PIGPM com moldagem no pré operatório e confecção de simples placa obturadora, ou apenas citações que realizaram as próteses com cirurgia de modelos. Não encontramos nenhum trabalho com moldagem durante o ato cirúrgico, confeccionando-se a porção maxilar e instalando-se a prótese ao final da cirurgia. Também não encontramos referência contrária à sua utilização ${ }^{6-16}$.

Analisando nossos dados, observamos quanto ao tempo de internação do paciente que, dependendo da extensão da cirurgia, maior é a permanência hospitalar. Pacientes que somente realizaram maxilectomia, permaneceram em média cinco dias (mínimo de dois, máximo 12). Observando que, sem prótese, o paciente necessitaria de cuidados especiais com a sonda naso enteral para a alimentação e para impedir possível obstrução da mesma, o paciente deveria permanecer internado com assistência especializada de enfermagem, dificilmente conseguida em casa.

No caso dos pacientes que, além da maxilectomia, realizaram exenteração de órbita, o período de internação aumenta para 6,25 dias (mínimo de dois, máximo 12). Para estes pacientes as considerações feitas são ainda mais importantes e podemos ver no quadro anexo $\mathrm{n}^{\circ} 1$, que um paciente permaneceu internado por 37 dias, não conseguindo habituar-se à prótese pela gravidade do caso.

Para os pacientes com maxilectomia, exenteração da órbita e craniotomia, a situação é, todavia, mais grave, com período de internação de 29 dias (mínimo de 18, máximo 40). Observamos nestes pacientes que um apresentou perturbação neurológica, passageira, após a cirurgia, conseguindo alimentar-se por via oral após 16 dias, sendo mantida a SNE por precaução. Este paciente teve alta hospitalar no $18^{\circ}$ dia. O outro apresentou complicações neurológicas, permanecendo internado na UTI, mas conseguiu se alimentar por via oral antes dos 40 dias (no prontuário não consta a data da $1^{\mathrm{a}}$ alimentação). Este paciente foi posteriormente internado, indo a óbito 82 dias após a $1^{a}$ operação, por problemas não relacionados ao procedimento. 
A PIGPM proporcionou também, a possibilidade dos pacientes deglutirem, alimentando-se por via oral, com a conseqüente retirada da sonda naso enteral em média com 3,3 dias. Apenas um paciente apresentou dificuldade inicial na deglutição, dificuldade esta superada gradativamente após alguns dias.

No referente à retirada da sonda naso enteral, a PIGPM possibilita aos pacientes deglutirem e se alimentarem pela boca, com a consequiente retirada da sonda naso enteral em média com 3,3 dias. Apenas um paciente apresentou dificuldade inicial na deglutição, dificuldade esta superada gradativamente em quatro dias.

Em relação à fonação hipernasalada, não podemos duvidar das vantagens do uso da PIGPM Verificamos que todos os pacientes tiveram pouca ou mínima nasalação. Ouvindo o paciente, sem a prótese e com a prótese, na simples contagem de números de 1 a 10 e na emissão de palavras ou frases notamos grande diferença. Após a reabilitação com a prótese permanente, melhor adaptada, a fonação tornou-se ainda mais inteligível.

Quanto à deformidade facial observamos também, de maneira objetiva, uma retração menor nos pacientes por nós atendidos do que nos pacientes que não realizaram a PIGPM. (Figuras 4 e 5)

Encontramos citações sobre PIGPM, porém, não encontramos descrições sobre a técnica de execução e resultados de sua utilização. Encontramos técnicas, sobre: prótese seccionada com o bulbo tripartido; utilização de silicone, utilização de material termoplástico; utilização da abertura nasal como meio de retenção $0^{2,17,20,24-26}$.

Após levantamento realizado no LILACS, Bireme, Pubmed e na Scielo não encontramos nenhuma referência à PIGPM, que permitisse a comparação de um trabalho com outros autores.
Podemos inferir mesmo, sem a utilização dos protocolos validados de qualidade de vida de pacientes submetidos a maxilectomia , ainda que, em virtude da utilização da PIGPM, o restabelecimento físico e psicológico, a reintegração às atividades profissionais e sociais do paciente, a melhora da qualidade de vida é evidente ${ }^{27}$.

Baseados em nossos resultados, concluímos que a PIGPM pós maxilectomia reduz o tempo para retirada da sonda naso-enteral e o tempo de internação hospitalar; possibilita a alimentação precoce e melhora as condições da fala; reduz a deformidade provocada pela retração cicatricial; reintegra precocemente o paciente às suas atividades profissionais e sociais devendo ser adotada como rotina.
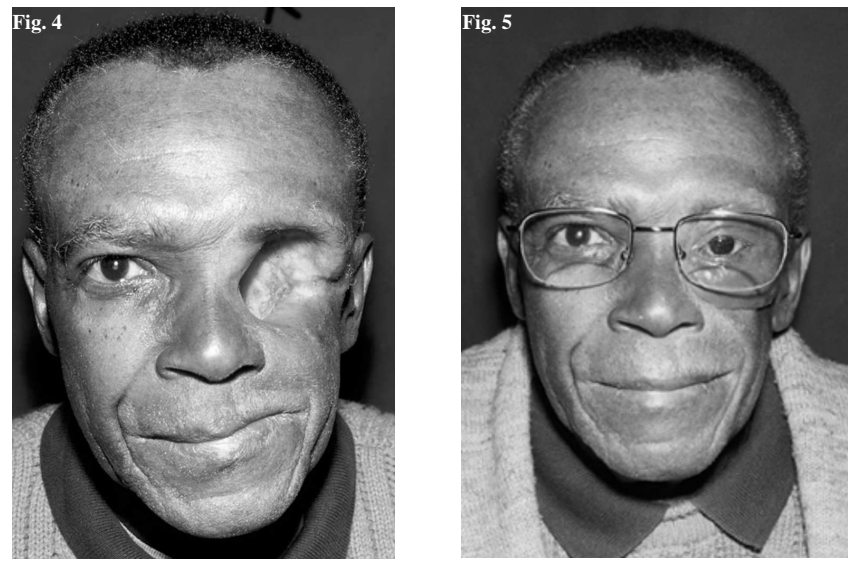

Figura 4 - Paciente submetido à maxilectomia e exenteração de órbita, com a utilização de prótese imediata, notar a reduzida deformidade na face (foto tirada sem a prótese).

Figura 5 - O mesmo paciente com a prótese restauradora. Notaro rosto praticamente sem deformação.

\begin{abstract}
Background: Evaluating the efficiency of the reconstruction with immediate prosthesis for large bone loss in patients submitted to maxillectomy considering: hospital outcome, taking out the nasoenteral tube, swallowing, speaking, funcional and esthetic reabilitation. Methods: We evaluated 20 patients, fourteen males and 6 females, with ages varying from 7 to 78 years old, eleven with squamous cell carcinoma. We performed the prosthesis modeling within the operation time with acrylic samples. The time of prosthesis modeling was about 45 minutes and all patients left the operating room with the prosthesis in their mouths. Results: The results were highly satisfactory for all patients. The hospital length of stay was 8.1 days; the nasoenteral tube was taken out in 7.3 days. The majority of patients had oral feeding in 3.3 days with paste food. The voice was considered good for all cases. Conclusion: We concluded that immediate prosthesis of large bone loss post maxillectomy reduced the hospital length of stay and the time of taking out the nasoenteral tube. The prosthesis allows earlier feeding and keeping the voice; reducing the cicatricial retraction and allowing the patient to return to his professional activities earlier. Therefore, we recommend that it must be done as a routine procedure.
\end{abstract}

Key words: Mouth neoplasms; Maxillofacial prosthesis; Denture, complete, immediate.

\section{REFERÊNCIAS}

1. Graziani M. Prótese maxilo facial. Rio de Janeiro: Científica; 1956.

2. Rezende JRV. Fundamentos da prótese buco-maxilo-facial. São Paulo: Sarvier; 1997.
3. Ali A, Fardy MJ, Patton DW. Maxillectomy--to reconstruct or obturate? Results of a UK survey of oral and maxillofacial surgeons. Br J Oral Maxillofac Surg. 1995;33(4):207-10.

4. Silva DP, Almeida FC, Vaccarezza GF, Brandão TB, Cazal C, Caroli A, Durazzo MD, Dias RB. Reabilitação protética de pacientes maxilectomizados. Uma contribuição das odontologia e um 
convite à reflexão. Pesq Bras Odontoped Clin Integr. 2004;4(2):125-30.

5. Keif F. Obturator prostheses for hemimaxillectomy patients. J Oral Rehabil. 2001;28(9):821-9.

6. MacCarthy D, Murphy N. Replacement of an obturator section of an existing two-piece implant-retained edentulous obturator. J Prosthet Dent. 2000;83(6):652-5.

7. Moseley HC, Horrocks EN, Welfare RR. Use of a modified twin block appliance following partial maxillectomy: case report. $\mathrm{Br}$ J Orthod. 1996;23(2):103-8.

8. Carvalho VA. Influência das próteses obturadoras e rebaixadoras de palato na terapia fonoaudiológica. In: Barros APB, Arakawa L, Tonini MO, Carvalho VA, orgs. Fonoaudiologia em cancerologia São Paulo: Fundação Oncocentro; 2000.

9. Haraguchi M, Mukohyama H, Taniguchi H. A simple method of fabricating an interim obturator prosthesis by duplicating the existing teeth and palatal form. J Prosthet Dent. 2006;95(6):46972.

10. Shaker KT. A simplified technique for construction of an interim obturator for a bilateral total maxillectomy defect. Int $\mathbf{J}$ Prosthodont. 2000;13(2):166-8.

11. Maire F, Kreher P, Toussaint B, Doliver G, Coffinet L. [Prosthesis fitting after maxillectomy an indispensable factor in acceptance and rehabilitation.] Rev Stomatol Chir Maxillofac. 2000;101(1):36-8.

12. Martin JW, Chambers MS, Lemon JC, Toth BB, Helfrick JF. Prosthodontic and surgical considerations for pediatric patiens requiring maxillectomy. Pediatr Dent. 1995;17(2):116-2.

13. Béneteau H, Crasson F, Labbé D, Riscala S, Alix T. Implants extra-oraux et irradiation: tendances actuelles. Rev Stomatol Chir Maxillofac. 2001;102(5):266-9.

14. Roumanas ED, Nishimura RD, Davis BK, Beumer J. Clinical evaluation of implants retaining edentulous maxillary obturator prostheses. J Prosthet Dent. 1997;77(2):184-90.

15. Har-El G, Bhaya M. Intraoperative fabrication of palatal prosthesis for maxillary resection. Arch Otolaryngol Head Neck Surg. 2001;28(7):834-6.

16. Cunningham R. A laboratory technique for the production of immediate surgical appliances and 'one stage' obturators for the hemi-maxillectomy patient. Br J Oral Maxillofac Surg. 1990;28(1):59-61.

17. Wang RR. Sectional prosthesis for total maxillectomy patients: a clinical report. J Prosthet Dent. 1997;78(3):241-4.

18. Dholam KP. Prosthetic rehabilitation of a patient following maxillectomy with orbital exenteration. Indian $\mathrm{J}$ Cancer. 2002;39(2):69-72.
19. Miracca RAA, Andrade Sobrinho J, Tanaka EMG. Magnetos na retenção de prótese conjugada óculo-palpebral e obturador palatino. PCL Rev Íbero Am Prótese Clínica \& Laboratorial. 2004;6(32):365-75.

20. Kanazawa T, Yoshida H, Furuya Y, Shimodaira K. Sectional prosthesis with hollow obturator portion made of thin silicone layer over resin frame. J Oral Rehabil. 2000;27(9):760-4.

21. Pigno MA, Funk JJ. Augmentation of obturator retention by extension into the nasal aperture: a clinical report. J Prosthet Dent. 2001;85(4):349-51.

22. Preiskel HW. Precision attachments in dentistry. St. Louis: Mosby; 1973.

23. Arigbede AO, Dosumu OO, Shaba OP, Esan TA. Evaluation of speech in patients with partial surgically acquired defects: pre and post prosthetic obturation. J Contemp Dent Pract. 2006;7(1):89-96.

24. Rieger J, Wolfaardt J, Seikaly H, Jha N. Speech outcomes in patients rehabilitated with maxillary obturator prostheses after maxillectomy: a prospective study. Int J Prostodont. 2002 MarApr;15(2):139-44.

25. Bueno YC, Curcio R. Reabilitação protética dos defeitos da cavidade oral. In: Carvalho MB. Tratado de cirurgia de cabeça e pescoço e otorrinolaringologia. São Paulo: Atheneu; 2001. p. 14059.

26. Didier M, Laccoureye O, Brasnu D, Vignon M. New surgical obturator prosthesis for hemimaxillectomy patients. J Prosthet Dent. 1993;69(5):520-3.

27. Rogers SN, Lowe D, McNally D, Brown JS, Vaughan ED. Healthrelated quality of life after maxillectomy: a comparison between prosthetic obturation and free flap. J Oral Maxillofac Surg. 2003;61(2):174-81.

Como citar este artigo:

Miracca RAR, Andrade Sobrinho J, Gonçalves AJ. Reconstrução com prótese imediata pós maxilectomia. Rev Col Bras Cir. [periódico na Internet]. [periódico na Internet] 2007 Set-Out; 34(3). Disponível em URL: http://www.scielo.br/rcbc

Endereço para correspondência:

Renato Alberto Aldo Miracca

Endereço: Rua Arthur Prado n 479 apto. 71

Paraíso

01322-000 - São Paulo - SP

E-mail-rmiracca@ig.com.br 